\title{
A Note on the Inequalities for Partial Affine System-Based Frames
}

\author{
Yu Tian* \\ College of Applied Sciences, Beijing University of Technology, Pingle Yuan 100, Beijing, 100124, P. R. China. \\ * Corresponding author. Tel.: +86-18101259982; email: yutian@emails.bjut.edu.cn \\ Manuscript submitted May 4, 2017; accepted July 15, 2017.
}

doi: 10.17706/ijapm.2017.7.4.284-290

\begin{abstract}
In this article, partial affine system-based frames is studied. We obtain some inequalities in form of matrix for a partial affine system to be a partial affine frame in the setting of subspaces of $L^{2}(\mathbb{R})$. The results improve known ones by W. Sun, Chui, Shi, and Chen, Ron and Shen.
\end{abstract}

Key words: Frame, wavelet frame, partial affine system.

\section{Introduction}

The frame was first introduced in 1952 by Duffin and Schaeffer [1] to study nonharmonic Fourier series and reintroduced in 1986 by Daubechies et al. [2]. Due to their potential applications in signal processing, filter theory and many other areas [3], [4], in recent years, frames have interested some mathematicians and engineering specialists. Among all forms of the frame, the wavelet frame is very important both in theory and in applications. The readers can refer to [5]-[13] for details.

Let $\psi \in L^{2}(\mathbb{R})$, and $\mathrm{a}>1$ be constant. Define $\psi_{j, k}=a^{\frac{j}{2}} \psi\left(a^{j} \cdot-k\right), j, k \in \mathbb{Z}$. If $\left\{\psi_{j, k}: j, k \in \mathbb{Z}\right\}$ forms a frame for $L^{2}(\mathbb{R})$, that is, there exist $0 \leq A \leq B<\infty$ such that

$$
A\|f\|^{2} \leq \sum_{j, k \in \mathbb{Z}}\left|\left\langle f, \psi_{j, k}\right\rangle\right|^{2} \leq B\|f\|^{2}, \quad \forall f \in L^{2}(\mathbb{R})
$$

then we call $\left\{\psi_{j, k}: j, k \in \mathbb{Z}\right\}$ is a wavelet frame. $A$ and $B$ are called the lower and upper frame bounds, respectively.

In applications, image denoising and data compression are two important tasks in signal analysis, and we often need to process signals with some limitations in time and frequency domain. Let us take wavelet frames expansion for example. For $\forall f \in L^{2}(\mathbb{R})$, we have the expansion

$$
f(\cdot)=\sum_{j, k \in \mathbb{Z}} c_{j, k} \psi_{j, k}(\cdot)
$$

they mean to set $c_{j, k}$ with large $j$ equal to zero (since large $j$ correspond to high-frequency) and then, for the left terms, to retain only large $c_{j, k}$ (in absolute value).

The above discussion inspired us to study the system of the form $X_{J}(\psi)=\left\{\psi_{j, k}: j \in J, k \in \mathbb{Z}\right\}$, where $J$ is a subset of $\mathbb{Z}$. We write $X_{\mathbb{Z}}(\psi)=X(\psi)$ for simplicity, and write 


$$
\Omega(J, \psi)=\left\{\xi \in \mathbb{R}: \sum_{j \in J}\left|\widehat{\psi}\left(a^{-j} \xi\right)\right|^{2}>0\right\}
$$

and $F L^{2}(E)=\left\{f \in L^{2}(\mathbb{R}): \hat{f}=0\right.$ a.e. on $\left.\mathbb{R} \backslash E\right\}$ for a Lebesgue measurable set $E$ in $\mathbb{R}$, where the Fourier transform of a function $f \in L^{2}(\mathbb{R})$ is defined by $\hat{f}(\cdot)=\int_{\mathbb{R}} f(x) e^{-2 \pi i x \cdot} d x$.

Since $J$ does not need to be $\mathbb{Z}$, we call $X_{J}(\psi)$ a partial affine system.

In the study of wavelet frames, one of the basic questions is to find conditions on $\psi$ and $a$ such that $\left\{\psi_{j, k}: j, k \in \mathbb{Z}\right\}$ forms a frame. Recently, many results including necessary and sufficient conditions have been established in $L^{2}(\mathbb{R})$ or in the subspaces of $L^{2}(\mathbb{R})$, for example, see [3], [6], [7], [10], [12], [13]-[16]. By a standard argument, for the wavelet frame in $F L^{2}(\Omega(J, \psi))$, we have the following necessary condition:

Proposition 1.1 Suppose that $X_{J}(\psi)$ is a frame for $F L^{2}(\Omega(J, \psi))$ with frame bounds $A$ and $B$, then

$$
A \chi_{\Omega(J, \psi)}(\cdot) \leq \sum_{j \in J}\left|\hat{\psi}\left(a^{-j} \cdot\right)\right|^{2} \leq B \chi_{\Omega(J, \psi)}(\cdot)
$$

a.e. on $\mathbb{R}$.

In this paper, we will generalize proposition 1.1, a necessary condition in form of matrix for a partial affine system to be a partial affine frame in $F L^{2}(\Omega(J, \psi))$ is obtained.

Throughout this paper, we denote by $\mathbb{C}$ and $\mathbb{Z}$ the set of complex number and the set of integers, respectively. Given a Lebesgue measurable set $E, E$ denotes the Lebesgue measure of $E$ and $\chi_{E}$ denotes the characteristic function on $E$. For each $f \in L^{2}(\mathbb{R})$, define $\operatorname{supp} f=\{x \in \mathbb{R}: f(x) \neq 0\}$, which is well-defined up to a set of measure zero.

Next we will give some notations. For a $>1$, denote $\Lambda_{J}=\left\{\alpha \in \mathbb{R}: \alpha=a^{j} k\right.$ for some $\left.j \in J, k \in \mathbb{Z}\right\}$. For any $\alpha \in \Lambda_{J}$, let $I_{J}(\alpha)=\left\{(j, k) \in J \times \mathbb{Z}: \alpha=a^{j} k\right\}$ and

$$
\Delta_{\alpha}(\psi, \omega)=\sum_{(j, k) \in I_{J}(\alpha)} \hat{\psi}\left(a^{-j} \omega\right) \overline{\hat{\psi}\left(a^{-\jmath} \omega+k\right)}
$$

For every $\mathrm{n} \geq 1$ and $\alpha \in \Lambda_{J} \backslash\{0\}$, let $G_{\alpha, n}(\psi, \omega)=\left[\Delta_{(k-m) \alpha}(\psi, \omega+m \alpha)\right]_{-n \leq k, m \leq n}$ be a $(2 n+1) \times$ $(2 n+1)$ matrix.

For an $n \times n$ Hermite matrix $G$ and constants $A$ and $B$, the inequalities $A \leq G \leq B$ stant for $A\|c\|^{2} \leq c^{T} G \bar{c} \leq B\|c\|^{2}, \quad \forall c \in \mathbb{C}^{n}$.

The rest of this paper is organized as follows. Section 2 is devoted to giving some lemmas. Section 3 focuses on the proof of our main result.

\section{Some Lemmas}

In this section, we give some Lemmas. Denote

$$
\mathcal{D}=\left\{f \in L^{2}(\mathbb{R}): \hat{f} \in L^{\infty}(\mathbb{R}) \text { and } \operatorname{supp} \hat{f}\left\{\omega \in \mathbb{R}: C^{-1} \leq|\xi| \leq C\right\} \text { for some } C>1\right\}
$$

We call a function $\psi \in \mathrm{L}^{2}(\mathbb{R})$ admissible if 


$$
C_{\psi}=\int_{\mathbb{R}} \frac{|\hat{\psi}(\omega)|^{2}}{|\omega|} d \omega<\infty
$$

It is easy to check that (1.2) implies the admissibility of $\psi$. The next Lemma is in [17, Lemma 2.1], which will be used in changing the order of the summation.

Lemma 2.1 For any $f \in \mathcal{D}$ and $\psi \in L^{2}(\mathbb{R})$ with $C_{\psi}<\infty$, we have

$$
\sum_{j, k \in \mathbb{Z}} \int_{-\infty}^{+\infty}\left|\hat{f}(\omega) \hat{f}\left(\omega+a^{j} k\right) \hat{\psi}\left(a^{-j} \omega\right) \hat{\psi}\left(a^{-j} \omega+k\right)\right| d \omega<\infty
$$

Lemma 2.2 Let $f, \psi \in L^{2}(\mathbb{R})$ and $j \in \mathbb{Z}$. Then for $k \in \mathbb{Z}$, the $k$-th Fourier coefficient of

$$
\sum_{k \in \mathbb{Z}} a^{\frac{j}{2}} \hat{f}\left(a^{j}(\cdot+k)\right) \overline{\hat{\psi}(\cdot+k)}
$$

is $\left\langle f, \psi_{j, k}\right\rangle$. In particular,

$$
\sum_{k \in \mathbb{Z}} a^{\frac{j}{2}} \hat{f}\left(a^{j}(\cdot+k)\right) \overline{\hat{\psi}(\cdot+k)}=\sum_{k \in \mathbb{Z}}\left\langle f, \psi_{j, k}\right\rangle e^{-2 \pi i}
$$

if $\left\{\left\langle f, \psi_{j, k}\right\rangle\right\}_{k \in \mathbb{Z}} \in l^{2}(\mathbb{Z})$.

Lemma 2.3 Let $\emptyset \neq K \subset \mathbb{Z}$, and $\psi \in L^{2}(\mathbb{R})$ with $C_{\psi}<\infty$. Then

$$
\sum_{j \in K} \sum_{k \in \mathbb{Z}}\left|\left\langle f, \psi_{j, k}\right\rangle\right|^{2}=\sum_{\alpha \in \Lambda_{K}} \sum_{(j, k) \in I_{K}(\alpha)} \int_{-\infty}^{+\infty} \overline{\hat{f}(\omega)} \hat{\psi}\left(a^{-j} \omega\right) \overline{\hat{\psi}\left(a^{-\jmath} \omega+k\right)} \hat{f}(\omega+\alpha) d \omega
$$

for $f \in \mathcal{D}$ and $\alpha \in \Lambda_{K}$

Proof. By Lemma 2.2

$$
\sum_{j \in K} \sum_{k \in \mathbb{Z}}\left|\left\langle f, \psi_{j, k}\right\rangle\right|^{2}=\sum_{j \in K} a^{j} \int_{0}^{1}\left|\sum_{k \in \mathbb{Z}} \hat{f}\left(a^{j}(\omega+k)\right) \overline{\hat{\psi}(\omega+k)}\right|^{2} d \omega .
$$

By a change of variables, we have

$$
\begin{aligned}
\sum_{j \in K} \sum_{k \in \mathbb{Z}}\left|\left\langle f, \psi_{j, k}\right\rangle\right|^{2} & =\sum_{j \in K} \int_{0}^{a^{j}}\left|\sum_{k \in \mathbb{Z}} \hat{f}\left(\omega+a^{j} k\right) \overline{\hat{\psi}\left(a^{-\jmath} \omega+k\right)}\right|^{2} d \omega \\
& =\sum_{j \in \mathrm{K}} \int_{0}^{a^{j}} \sum_{k \in \mathbb{Z}} \overline{\hat{f}\left(\omega+a^{J} k\right)} \hat{\psi}\left(a^{-j} \omega+k\right) \cdot F(\omega) d \omega,
\end{aligned}
$$

where

$$
F(\omega)=\sum_{k \in \mathbb{Z}} \hat{f}\left(\omega+a^{j} k\right) \overline{\hat{\psi}\left(a^{-\jmath} \omega+k\right)} .
$$


Since $f \in \mathcal{D}$, so for each $j \in K$, the number of $k$ for which $\hat{f}\left(\omega+a^{j} k\right) \neq 0$ is finite, then

$$
\sum_{j \in K} \sum_{k \in \mathbb{Z}}\left|\left\langle f, \psi_{j, k}\right\rangle\right|^{2}=\sum_{j \in K} \int_{-\infty}^{+\infty} \overline{\hat{f}(\omega)} \hat{\psi}\left(a^{-j} \omega\right) \sum_{k \in \mathbb{Z}} \hat{f}\left(\omega+a^{j} k\right) \overline{\hat{\psi}\left(a^{-\jmath} \omega+k\right)} d \omega
$$

By Lemma 2.1, we can change the summation order, so

$$
\begin{aligned}
\sum_{j \in K} \sum_{k \in \mathbb{Z}}\left|\left\langle f, \psi_{j, k}\right\rangle\right|^{2} & =\sum_{j \in K} \sum_{k \in \mathbb{Z}} \int_{-\infty}^{+\infty} \overline{\hat{f}(\omega)} \hat{f}\left(\omega+a^{j} k\right) \hat{\psi}\left(a^{-j} \omega\right) \overline{\hat{\psi}\left(a^{-\jmath} \omega+k\right)} d \omega \\
& =\sum_{\alpha \in \Lambda_{K}} \sum_{(j, k) \in I_{K}(\alpha)} \int_{-\infty}^{+\infty} \overline{\hat{f}(\omega)} \hat{\psi}\left(a^{-j} \omega\right) \overline{\hat{\psi}\left(a^{-\jmath} \omega+k\right) \hat{f}(\omega+\alpha) d \omega}
\end{aligned}
$$

This finishes the proof.

\section{Proof of the Main Result}

In this section, we give the main result and prove it.

Our main result is the theorem below, which is a generalization of [17, Theorem 1.2]:

Theorem 1.1 Let $\boldsymbol{\psi} \in \boldsymbol{L}^{2}(\mathbb{R})$ and $\boldsymbol{a}>\mathbf{1}$ be constants. If $\boldsymbol{X}_{\boldsymbol{J}}(\boldsymbol{\psi})$ is a frame for $\boldsymbol{F} \boldsymbol{L}^{2}(\boldsymbol{\Omega}(J, \boldsymbol{\psi}))$ with frame bounds $\boldsymbol{A}$ and $\boldsymbol{B}$. Then

$$
\mathrm{A} \chi_{\Omega(J, \psi)} \leq \boldsymbol{G}_{\boldsymbol{\alpha}, \boldsymbol{n}}(\boldsymbol{\psi}, \boldsymbol{\omega}) \leq \mathrm{B} \chi_{\Omega(J, \psi),} \text { a.e., } \forall \boldsymbol{n} \geq \mathbf{1}, \boldsymbol{\alpha} \in \Lambda_{J} \backslash\{\mathbf{0}\}
$$

Proof. Fix some $\boldsymbol{\alpha} \in \boldsymbol{\Lambda}_{\boldsymbol{J}} \backslash\{\boldsymbol{0}\} . \sum_{j \in J}\left|\widehat{\boldsymbol{\psi}}\left(\boldsymbol{a}^{-j} \boldsymbol{\omega}\right)\right|^{2}$ and $\sum_{(j, s) \in I_{J}(\boldsymbol{\alpha})} \widehat{\boldsymbol{\psi}}\left(\boldsymbol{a}^{-j} \boldsymbol{\omega}\right) \overline{\widehat{\boldsymbol{\psi}}\left(\boldsymbol{a}^{-J}(\boldsymbol{\omega}+\boldsymbol{\alpha})\right)}$ are locally integrable on $\mathbb{R}$. Let

$$
\begin{aligned}
\Gamma=\bigcap_{M \in \mathbb{Z},-n \leq m \leq n} & \left\{\omega: \omega \text { is a Lebesgue point for } \sum_{j \in[-M+1, \infty) \cap J}\left|\hat{\psi}\left(a^{-j}(\omega+m \alpha)\right)\right|^{2}, \sum_{j \in[-M+1, \infty) \cap J} \mid \hat{\psi}\left(a^{-j}(\omega\right.\right. \\
& +m \alpha))\left.\right|^{2} \text { and } \sum_{\substack{(j, s) \in I_{J}(\alpha) \\
j>-M}} \hat{\psi}\left(a^{-j}\left(\omega_{0}+k \alpha\right) \overline{\left(\left(a^{-J}(\omega+k \alpha)+s\right)\right.},|k| \leq n \text {, respectively }\right\} .
\end{aligned}
$$

Then $\mathbb{R} \backslash \boldsymbol{\Gamma}$ is a zero-measured set.

Fix any $\boldsymbol{\omega}_{\mathbf{0}}$ in $\boldsymbol{\Gamma}$ such that $\boldsymbol{\omega}_{\mathbf{0}}+\boldsymbol{m} \boldsymbol{\alpha} \neq \mathbf{0},-\boldsymbol{n} \leq \boldsymbol{m} \leq \boldsymbol{n}$. For $\mathbf{0}<\boldsymbol{\varepsilon}<|\boldsymbol{\alpha}|, \boldsymbol{\beta} \in \mathbb{R}$ and $\mathbf{c}=\left\{\boldsymbol{c}_{m}\right\}_{-n \leq m \leq n} \in \mathbb{C}^{2 n+1}$, denote $\boldsymbol{H}_{\varepsilon}(\boldsymbol{\beta})=\left[\boldsymbol{\omega}_{0}+\boldsymbol{\beta}-\frac{\varepsilon}{2}, \boldsymbol{\omega}_{0}+\boldsymbol{\beta}+\frac{\varepsilon}{2}\right]$ and

$$
\hat{g}_{\varepsilon}(\omega)=\frac{1}{\sqrt{\varepsilon}} \sum_{m=-n}^{n} c_{m} \chi_{H_{\varepsilon}(m \alpha)}(\omega) \chi_{\Omega(J, \psi)}(\omega) .
$$

Fix some $M \in \mathbb{N}$, and write 


$$
\sum_{j \in J} \sum_{k \in \mathbb{Z}}\left|\left\langle g_{\varepsilon}, \psi_{j, k}\right\rangle\right|^{2}=\sum_{j \in(-\infty-M] \cap J} \sum_{k \in \mathbb{Z}}\left|\left\langle g_{\varepsilon}, \psi_{j, k}\right\rangle\right|^{2}+\sum_{j \in[-M+1, \infty) \cap J} \sum_{k \in \mathbb{Z}}\left|\left\langle g_{\varepsilon}, \psi_{j, k}\right\rangle\right|^{2}=Q_{1}+Q_{2}
$$

By Lemma 2.3

$$
Q_{2}=\sum_{j \in[-M+1, \infty) \cap j} \sum_{s \in \mathbb{Z}} \int_{-\infty}^{+\infty} \overline{\hat{g}_{\varepsilon}(\omega)} \hat{g}_{\varepsilon}\left(\omega+a^{j} s\right) \hat{\psi}\left(a^{-j} \omega\right) \overline{\hat{\psi}\left(a^{-j} \omega+s\right)} d \omega
$$

Since $X_{J}(\psi) \subset F L^{2}(\Omega(J, \psi))$, so $\chi_{\Omega(J, \psi)}(\cdot) \hat{\psi}\left(a^{-j} \cdot\right)=\hat{\psi}\left(a^{-j} \cdot\right)$, then we have

$$
Q_{2}=\sum_{j \in[-M+1, \infty) \cap} \sum_{s \in \mathbb{Z}} \int_{-\infty}^{+\infty} \overline{\hat{h}_{\varepsilon}(\omega)} \hat{h}_{\varepsilon}\left(\omega+a^{j} s\right) \hat{\psi}\left(a^{-j} \omega\right) \overline{\hat{\psi}\left(a^{-\jmath} \omega+s\right)} d \omega
$$

where

$$
\hat{h}_{\varepsilon}(\omega)=\frac{1}{\sqrt{\varepsilon}} \sum_{m=-n}^{n} c_{m} \chi_{H_{\varepsilon}(m \alpha)}(\omega)
$$

Then by the same procedure in [17], we can obtain

$$
\begin{aligned}
& \sum_{\alpha^{\prime} \in \Lambda_{J}(j, s) \in I_{J}\left(\alpha^{\prime}\right)} \int_{\mathbb{R}} \overline{\hat{h}_{\varepsilon}(\omega)} \hat{h}_{\varepsilon}\left(\omega+\alpha^{\prime}\right) \hat{\psi}\left(a^{-j} \omega\right) \overline{\hat{\psi}\left(a^{-\jmath} \omega+s\right)} d \omega \\
& =\sum_{\substack{(j, s) \in I_{J}(0) \\
j>-M}} \int_{\mathbb{R}}\left|\hat{h}_{\varepsilon}(\omega)\right|^{2} \cdot\left|\hat{\psi}\left(a^{-j} \omega\right)\right|^{2} d \omega \\
& +\sum_{m=1}^{2 n} \sum_{\substack{(j, s) \in I_{J}(m \alpha) \\
j>-M}} \int_{\mathbb{R}} \overline{\hat{h}_{\varepsilon}(\omega)} \hat{h}_{\varepsilon}(\omega+m \alpha) \hat{\psi}\left(a^{-j} \omega\right) \overline{\hat{\psi}\left(a^{-J} \omega+s\right)} d \omega \\
& +\sum_{m=1}^{2 n} \sum_{\substack{(j, s) \in I_{J}(-m \alpha) \\
j>-M}} \int_{\mathbb{R}} \overline{\hat{h}_{\varepsilon}(\omega)} \hat{h}_{\varepsilon}(\omega-m \alpha) \hat{\psi}\left(a^{-j} \omega\right) \overline{\hat{\psi}\left(a^{-J} \omega+s\right)} d \omega \\
& +\sum_{\alpha^{\prime} \in \Lambda_{J} \backslash\{ \pm m \alpha, 0 \leq m \leq 2 n\}} \sum_{\substack{(j, s) \in I_{J}\left(\alpha^{\prime}\right) \\
j>-M}} \int_{\mathbb{R}} \overline{\hat{h}_{\varepsilon}(\omega)} \hat{h}_{\varepsilon}\left(\omega+\alpha^{\prime}\right) \hat{\psi}\left(a^{-j} \omega\right) \overline{\hat{\psi}\left(a^{-J} \omega+s\right)} d \omega \\
& =Q_{2,1}+Q_{2,2}+Q_{2,3}+Q_{2,4} \text {, }
\end{aligned}
$$

and we also have

$$
\begin{gathered}
\lim _{\varepsilon \rightarrow 0} \sup \left|Q_{1}\right| \leq C^{\prime} \sum_{m=-n}^{n} \int_{|\omega|>2 a^{M}\left|\omega_{0}+m \alpha\right| /(a+1)}|\hat{\psi}(\omega)|^{2} d \omega \\
+C^{\prime} \sum_{m=-n}^{n} \sum_{j \in(-\infty-M] \cap J}\left|\hat{\psi}\left(a^{-j}\left(\omega_{0}+m \alpha\right)\right)\right|^{2}
\end{gathered}
$$




$$
\begin{gathered}
\lim _{\varepsilon \rightarrow 0} Q_{2,1}=\lim _{\varepsilon \rightarrow 0} \sum_{\substack{(j, s) \in I_{J}(0) \\
j>-M}} \frac{1}{\varepsilon} \sum_{k=-n}^{n}\left|c_{k}\right|^{2} \int_{\omega_{0}+k \alpha-\frac{\varepsilon}{2}}^{\omega_{0}+k \alpha+\frac{\varepsilon}{2}}\left|\hat{\psi}\left(a^{-j} \omega\right)\right|^{2} d \omega \\
=\sum_{k=-n}^{n}\left|c_{k}\right|^{2}\left(\sum_{j \in[-M+1, \infty) \cap J}\left|\hat{\psi}\left(a^{-j}\left(\omega_{0}+k \alpha\right)\right)\right|^{2}\right) \\
\lim _{\varepsilon \rightarrow 0} Q_{2,2}=\sum_{m=1}^{2 n} \sum_{k=-n}^{n-m} \overline{c_{k}} c_{k+m} \sum_{\substack{(j, s) \in I_{J}(m \alpha) \\
j>-M}} \hat{\psi}\left(a^{-j}\left(\omega_{0}+k \alpha\right)\right) \bar{\psi}\left(a^{-J}\left(\omega_{0}+k \alpha\right)+s\right) \\
\lim _{\varepsilon \rightarrow 0} Q_{2,3}=\sum_{m=1}^{2 n} \sum_{k=m-n}^{n} \overline{c_{k}} c_{k-m} \sum_{\substack{(j, s) \in I_{J}(-m \alpha) \\
j>-M}} \hat{\psi}\left(a^{-j}\left(\omega_{0}+k \alpha\right)\right) \overline{\hat{\psi}\left(a^{-J}\left(\omega_{0}+k \alpha\right)+s\right)} \\
\lim _{\varepsilon \rightarrow 0} Q_{2,4}=0
\end{gathered}
$$

Combining (3.1)-(3.5), we obtain

$$
\begin{aligned}
& \left.\lim _{\varepsilon \rightarrow 0} \sup \left|Q_{1}+Q_{2}-\sum_{k=-n}^{n}\right| c_{k}\right|^{2}\left(\sum_{j \in[-M+1, \infty) \cap J}\left|\hat{\psi}\left(a^{-j}\left(\omega_{0}+k \alpha\right)\right)\right|^{2}\right)- \\
& -\sum_{m=1}^{2 n} \sum_{k=-n}^{n-m} \overline{c_{k}} c_{k+m} \sum_{\substack{(j, s) \in I_{J}(m \alpha) \\
j>-M}} \hat{\psi}\left(a^{-j}\left(\omega_{0}+k \alpha\right)\right) \overline{\hat{\psi}\left(a^{-J}\left(\omega_{0}+k \alpha\right)+s\right)} \\
& -\sum_{m=1}^{2 n} \sum_{k=m-n}^{n} \overline{c_{k}} c_{k-m} \sum_{\substack{(j, s) \in I_{J}(-m \alpha) \\
j>-M}} \hat{\psi}\left(a^{-j}\left(\omega_{0}+k \alpha\right)\right) \overline{\hat{\psi}\left(a^{-\jmath}\left(\omega_{0}+k \alpha\right)+s\right)} \mid \\
& \leq C^{\prime} \sum_{m=-n}^{n} \int_{|\omega|>2 a^{M}\left|\omega_{0}+m \alpha\right| /(a+1)}|\widehat{\psi}(\omega)|^{2} d \omega+C^{\prime} \sum_{m=-n}^{n} \sum_{j \in(-\infty-M] \cap J}\left|\widehat{\psi}\left(a^{-j}\left(\omega_{0}+m \alpha\right)\right)\right|^{2} .
\end{aligned}
$$

By letting $M \rightarrow \infty$, we have

$$
\begin{aligned}
\lim _{\varepsilon \rightarrow 0}\left(Q_{1}+Q_{2}\right)= & \sum_{k=-n}^{n}\left|c_{k}\right|^{2} \Delta_{0}\left(\psi, \omega_{0}+k \alpha\right) \\
& +\sum_{m=1}^{2 n}\left[\sum_{k=m-n}^{n} \overline{c_{k}} c_{k-m} \Delta_{m \alpha}\left(\psi, \omega_{0}+k \alpha\right)+\sum_{k=m-n}^{n} \overline{c_{k}} c_{k-m} \Delta_{-m \alpha}\left(\psi, \omega_{0}+k \alpha\right)\right] .
\end{aligned}
$$

Since $\left\|\hat{g}_{\varepsilon}\right\|^{2}=\|c\|^{2} \chi_{\Omega(J, \psi)}$, it follows that

$$
\begin{aligned}
A \chi_{\Omega(J, \psi)}\|c\|^{2} \leq & \sum_{k=-n}^{n}\left|c_{k}\right|^{2} \Delta_{0}\left(\psi, \omega_{0}+k \alpha\right) \\
& +\sum_{m=1}^{2 n}\left[\sum_{k=m-n}^{n} \overline{c_{k}} c_{k-m} \Delta_{m \alpha}\left(\psi, \omega_{0}+k \alpha\right)+\sum_{k=m-n}^{n} \overline{c_{k}} c_{k-m} \Delta_{-m \alpha}\left(\psi, \omega_{0}+k \alpha\right)\right] \leq B \chi_{\Omega(J, \psi)}\|c\|^{2} .
\end{aligned}
$$

Since $\mathrm{c}$ is arbitrary, the above inequalities are equivalent to $\mathrm{A} \chi_{\Omega(J, \psi)} \leq G_{\alpha, n}(\psi, \omega) \leq \mathrm{B} \chi_{\Omega(J, \psi)}$. This 
completes the proof.

\section{Conclusion}

In this paper, we obtain some inequalities in form of matrix for a partial affine system to be a partial affine frame in the setting of subspaces of $L^{2}(\mathbb{R})$.

\section{Acknowledgment}

This work was supported by National Natural Science Foundation of China (Grant No. 11271037).

\section{References}

[1] Duffin, R. J. \& Schaeffer, A. C. (1952). A class of nonharmonic Fourier series. Trans. Amer. Math. Soc., 72, 341-366.

[2] Daubechies, I., Grossmann, A., \& Meyer, Y. (1986). Painless nonorthogonal expansions. Journal of Mathematical Physics, 27, 1271-1283.

[3] Christensen, O. (2016). An introduction to Frames and Riesz Bases. Boston: Birkhäuser.

[4] Young, R. (1990). An Introduction to Non-harmonic Fourier Series. New York: Academic Press.

[5] Bownik, M. (2000). A characterization of affine dual frames in $L^{2}\left(\mathbb{R}^{n}\right)$. Appl. Comput. Harmon. Anal., 8, 203-221.

[6] Chui, C. K., \& Shi, X. L. (2000) Orthonormal wavelets and tight frames with arbitrary dilations. Appl. Comput. Harmon. Anal., 9, 243-264.

[7] Chui, C. K., \& Shi, X. L. (1993). Inequalities of Littlewood-Paley type for frames and wavelets. SIAM J. Math. Anal., 24, 263-277.

[8] Dai, X., Diao, Y. \& Gu, Q. (2001). Subspaces with normalized tight frame wavelets in $\mathbb{R}$. Proceedings of Amer. Math. Soc., 130, 1661-1667.

[9] Dai, X., Diao, Y., Gu, Q., \& Han, D. (2003). The existence of subspace wavelet sets. J. Comput. Appl. Math., $155,83-90$.

[10] Han. B. (1997). On dual wavelet tight frames. Appl. Comput. Harmon. Anal., 4, 380-413.

[11] Jia, H.-F., \& Li, Y.-Z. (2015). Weak (quasi-)affine bi-frames for reducing subspaces of $L^{2}\left(\mathbb{R}^{d}\right)$. Sci. China Math., 58, 1005-1022.

[12] Ron, A., \& Shen, Z. (1997). Affine systems in $L^{2}\left(\mathbb{R}^{d}\right)$ : The analysis of the analysis operator. J. Funct. Anal., $148,408-447$.

[13] Ron, A., \& Shen, Z. (2005).Generalized shift invarient systems. Constr. Approx., 22, 1-45.

[14] Daubechies, I. (1992). Ten Lectures on Wavelets. Philadelphia: SIAM.

[15] Daubechies, I. (1990). The wavelet transform, time-frequency localization and signal analysis. IEEE Trans Inform Theory, 36, 961-1005.

[16] Shi, X. L., \& Chen, F. (2005). Necessary condition and sufficient condition for affine frames. Since in China, Ser. A., 48, 1369-1378.

[17] Sun, W., \& Zang, L. (2010). Inequalities for wavelet frames. Nume. Funct. Anal. Optim., 31, 1090-1101.

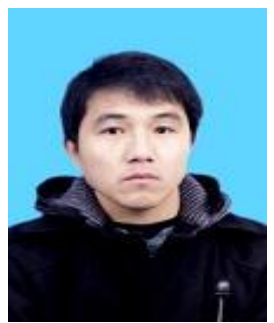

Yu Tian received his baccalaureate from College of Mathematical Sciences, Shangqiu Normal University He Nan, P. R. China in 2012. He is now pursuing the Ph.D degree in College of Applied Sciences, Beijing University of Technology, Beijing, P. R. China. His research interests include wavelet analysis and frame theory. 